\title{
A Novel Prioritization Scheme to Improve QoS in IEEE 802.11e Networks
}

\author{
Navid Tadayon, Saadan Zokaei, and Elaheh Askari \\ The Electrical Engineering Department, K. N. Toosi University of Technology, Seyed khandan, \\ Dr. Shariati Ave, P.O. Box 16315-1355, Tehran, Iran \\ Correspondence should be addressed to Navid Tadayon, navid.tadayon@gmail.com
}

Received 8 October 2009; Revised 6 March 2010; Accepted 21 March 2010

Academic Editor: Sghaier Guizani

Copyright ( 2010 Navid Tadayon et al. This is an open access article distributed under the Creative Commons Attribution License, which permits unrestricted use, distribution, and reproduction in any medium, provided the original work is properly cited.

IEEE 802.11 WLAN utilizes a distributed function at its MAC layer, namely, DCF to access the wireless medium. Due to its distributed nature, DCF is able to guarantee working stability in a wireless medium while maintaining the assembling and maintenance cost in a low level. However, DCF is inefficient in dealing with real-time traffics due to its incapability on providing QoS. IEEE 802.11e was introduced as a supplementary standard to cope with this problem. This standard introduces an Enhanced Distributed Coordination Function (EDCF) that works based on diff-Serve model and can serve multiple classes of traffics (by using different prioritizations schemes). With the emergence of new time-sensitive applications, EDCF has proved to be yet inefficient in dealing with these kinds of traffics because it could not provide network with well-differentiated QoS. In this study, we propose a novel prioritization scheme to improve QoS level in IEEE 802.11e network. In this scheme, we replace Uniform PDF with Gamma PDF, which has salient differentiating properties. We investigate the suitability and superiority of this scheme on furnishing network with well-differentiated QoS using probabilistic analysis. We strengthen our claims by extensive simulation runs.

\section{Introduction}

IEEE 802.11 [1-3] stations utilize two different functions at its MAC layers to access the radio channel; the first one is DCF, which is the mandatory part, and the second one is an optional function named Point Coordination Function (PCF). DCF works based on Carrier Sense Multiple Access with Collision Avoidance (CSMA/CA), and as will be explained later it uses a binary exponential back-off mechanism for transmission. However, DCF has a very restricted potential in supporting multimedia traffic with different QoS requirements. On the other side, PCF can supply network with a minimum amount of QoS although it might not be implemented in most of network cards due to its complexity, inefficiency, and instability issues.

In DCF access mechanism, the station that is going to transmit should firstly sense the channel. This action should be continued until the channel becomes ideal and remains ideal for at least DIFS. After spending this time, station should wait for a random amount of time to be expired before it starts to transmit its frame. This time is known as back-off duration to which a back-off counter is dedicated. Back-off counters decrease their values during channel ideal bursts and stop counting down as soon as channel becomes busy. The countdown process activates again, upon channel becomes ideal, and remains ideal for at least DIFS duration. Finally, the station transmits unconditionally as soon as the counter value reaches zero.

These back-off durations are to be chosen uniformly from the interval $\left[0, \mathrm{CW}_{i}-1\right]$ in which $\mathrm{CW}_{i}$ is the current contention window's length. At the first attempt to transmit, $\mathrm{CW}_{i}$ is always equal to initial contention window's size $\left(\mathrm{CW}_{\min }\right)$. After each unsuccessful transmission of a frame, $\mathrm{CW}_{i}$ gets larger (multiplied by a factor of 2). This process resumes until $\mathrm{CW}_{i}$ reaches $\mathrm{CW}_{\max }$ limit and then it stays unchanged until back-off stages reaches to $\mathrm{L}_{i}$, retry.

The station should reset its contention window's length in only two situations: first, when a frame is sent successfully; second, when retransmission counter reaches $\mathrm{L}_{i}$, retry and the frame drops subsequently.

After each successful reception of a frame, receiver should send a short control frame, named Ack, back to 
transmitter. In case the transmitter does not receive this controlling frame within a prespecified amount of time, namely, Ack-timeout, or hears different transmission from another station, it will reschedule its retransmission for a latter time. This retransmission has to be performed using back-off mechanism. This is named the Basic transmission mechanism.

In order to reduce the undesirable effects of hidden terminal problem, which is a common situation in many WLAN scenarios, another mechanism named RTS/CRS is applicable. RTS/CTS is a four-way handshake transmission mechanism.

So far, many researchers have focused on evaluating performance of IEEE 802.11 networks [4-15]. Bianchi in [4] introduced a simple and precise analytical model to compute network's throughput (in saturation condition). Ziouva and Antonakopoulos (ZAs) in [5] proposed an improved analytical model from Bianchi's one and extended this model to derive an expression for delivery-delay in 802.11 networks (in saturation condition). Wu et al. [6] improved Bianchi's model considering retry limit, which is one of important parameters in DCF. Xiao and Rosdahl in [7] directed a deep investigation on maximum network throughput. However, no one of these models (see [4-7]) inspected prioritization issue in network.

In DCF, stations should wait for a long time before starting to transmit. This fact causes many problems when we are to encounter with delay sensitive and time bounded traffics like voice and video [16]. An excellent solution to this problem is to use prioritization schemes instead of guaranteed methods of QoS provisioning (that is always an intricate solution to be implemented). By applying little changes on DCF, Priority schemes are simply implementable and could fall thoroughly effective to serve multiple traffic classes, concurrently [17]. Up to now, several researches have focused on applying differentiation over DCF. Deng and Cheng in [16] proposed a novel scheme by differentiating back-off window's length; in this scheme, the higher priority class uses $\left[0,2^{j+1}-1\right]$ interval and the lower priority class uses $\left[2^{j+1}, 2^{j+2}-1\right]$ interval for transmission $(j$ is the back-off stage in network). Veres et al. in [18] proposed a priority scheme in which differentiation applied on both initial back-off window's length $\left(\mathrm{CW}_{\min }\right)$ and maximum back-off window's length $\left(\mathrm{CW}_{\max }\right)$. Aad and Castelluccia in [19] presented another priority scheme that focused on Inter Frame Space prioritization. Pallot and Miller in [20] proposed a new prioritization scheme in which different traffic classes use different probability distribution functions (nonuniform) for their differentiation purpose. The above article briefly pointed out to making use of exponential distribution instead of Uniform one, in a network consisting of only two priority classes, and the authors refrained to give more details. In addition, authors did not point out to the probabilistic and mathematical analysis of their suggested scheme. Henceforth, in this article we thoroughly investigate this subject and strengthen our claims with probabilistic analysis and extensive simulations. Xiao in [17] has recently developed Bianchi's analytical model to evaluate the effect of all back-off priority schemes in one article. He evaluated differentiation resulting from $\mathrm{CW}_{\min }$, Persistent Factor (PF) and maximum back-off stage. IEEE 802.11 committee has recently approved another standard, namely, IEEE 802.11e [1] in order to support the QoS issue in MAC layer. The MAC layer in this standard uses a different access function named Hybrid Coordination Function (HCF). HCF includes two different access functions, the first one is EDCF, and the second one is a contention free centrally controlled function. EDCF makes use of priority schemes mentioned above to support QoS. Mangold et al. in [21] directed a performance study on EDCF and established extensive simulations. Xiao in [5] provided a comprehensive review of 802.11e networks.

This article organized as follows: following this section, we introduce common prioritization schemes implemented in EDCF so far. In Section 2, IEEE 802.11e existing analytical models will be discussed, and some of the network metrics will be introduced. In Section 3, our novel priority scheme based on PDF differentiation will be presented and in Section 4, performance of this scheme will be evaluated using extensive simulation runs. In Section 5, we will set up a comparison between scenarios using different well-known PDFs at their MAC layer. Finally, in Section 6, we conclude and summarize our study.

1.1. Prioritization Schemes in EDCF. In general, all priority schemes are put under one of these three categories: Back-off priority schemes [16-18], IFS priority schemes $[19,20]$, and Hybrid prioritization schemes [21-24].

Back-off priority schemes include

(1) differentiation on $\mathrm{CW}_{\min }[17,18]$,

(2) differentiation on PF [17],

(3) differentiation on $\mathrm{CW}_{\max }[18]$,

(4) differentiation on Probability Distribution [16, 20],

(5) differentiation on maximum back-off stages [17],

(6) combinations of above schemes [17, 18].

It should be noted that scheme (3) is directly derivable from (1), (2), and (5) in combination and should not be regarded as an independent scheme. In recent years, different aspects of IFS schemes have been studied and all back-off priority schemes except (4) have been under deep investigation. Therefore, in this article we direct a thorough analysis on (4). The interested readers are referred to $[25,26]$ to study more about IFS priority schemes.

Let us suppose that $\mathrm{N}$ different traffic classes exist $(i=$ $0 \ldots N-1)$. Following, we briefly introduce two of the most common priority schemes that are applied to EDCF, thus far.

(i) Back-off priority schemes: suppose that $\mathrm{CW}_{i, 0}, \sigma_{i}$, and $\mathrm{L}_{i \text {,retry, }}$, respectively represent contention window's length, persistent factor, and retry limit in class $i$. If class $i$ enjoys higher priority than class $j$, then at least one of these equalities should be strictly satisfied: $0 \leq \sigma_{i}<\sigma_{j}, \mathrm{CW}_{i, 0}<$ $\mathrm{CW}_{j, 0}, \mathrm{~L}_{i, \text { retry }}<\mathrm{L}_{j, \text { retry }}$.

To gain a better differentiation, we may combine more than one of the above parameters. Suppose that $\mathrm{CW}_{i, j}$ represents contention window's length at $j$ th stage and $\mathrm{CW}_{i, \max }$ represents maximum contention window's length. 
Then the following equations are true between introduced parameters and we will use them in the following analysis:

$$
=\left\{\begin{array}{rr}
\sigma_{i, j}^{j} \cdot \mathrm{CW}_{i, 0} & j=0 \ldots m_{i}-1 \text { when } \mathrm{L}_{i, \text { retry }} \geq m_{i} \\
\sigma^{m_{i}}{ }_{i} \cdot \mathrm{CW}_{i, 0}=\mathrm{CW}_{i, \text { max }} & j=m_{i} \ldots \mathrm{L}_{i \text {,retry when } \mathrm{L}_{i, \text { retry }} \geq m_{i}} \\
\sigma^{j}{ }_{i} \cdot \mathrm{CW}_{i, 0} & j=0 \ldots \mathrm{L}_{i, \text { retry }} \text { when } \mathrm{L}_{i, \text { retry }} \leq m_{i}
\end{array}\right\},
$$

(ii) IFS priority schemes: here, instead of using same IFS in all classes, an Arbitration Interframe Space (AIFS[i]) is utilized for each class. To that end, if $i$ has higher priority than $j$, then $\operatorname{AIFS}[i]<\operatorname{AIFS}[j]$.

\section{IEEE 802.11e Network Analytic Model and Modified Markov Chain}

Now, it is time to take a look at one of the existing analytical models of IEEE 802.11e networks.

\subsection{Analytic Model.}

(i) Suppose that, for a station belonging to class i $(i=$ $0 \ldots N-1)$, stochastic processes $\mathrm{b}(i, t)$ and $\mathrm{s}(i, t)$, represent, respectively, the back-off counter's value and the back-off stage at time $t$. Assume that pi symbolizes the collision probability of a transmitted frame from a station (belonging to class i) in a given timeslot. Also, $p_{b, i}$ symbolizes the channel busyness probability in a given timeslot from a viewer station's viewpoint (belonging to class i).

The transition state diagram for a class i transmission entity is depicted in Figure 1.

We applied several modifications on this model that were not considered in ZAs [5] and Bianchi's [4] models; for instance, we have the following.

(i) Our model is suitable for differentiation analysis on 802.11 networks.

(ii) Our model considers a limited $\mathrm{L}_{i}$, retry whereas in $[4$, 5] $\mathrm{L}_{i, \text { retry }}=\infty$.

(iii) Our model takes into account the counter freezing mechanism in EDCF that was not considered in [4].

(iv) Our model considers frame-dropping probability whereas no other model did.

Imagine $S_{i, j, k_{t \rightarrow \infty}}=\operatorname{Lim}(b(i, t)=j, s(i, t)=k)$; then in Figure 1, state probabilities are directly derivable from the above chain.

Transmission probability $\left(\tau_{i}\right): \tau_{i}$ represents the transmission probability in a given timeslot by a station belonging to class i. Each station starts to transmit, whenever its back-off counter value reaches zero, meaning that being in one of $S_{i, j, 0}$ states in Figure 1. Therefore, it is equal to

$$
\tau_{i}=\sum_{j=0}^{L_{i, \text { retry }}} S_{i, j, 0}=S_{i, 0,0} \cdot \frac{1-p_{i}^{L_{i, \text { retry }}} .}{1-p_{i}} .
$$

(i) Collision Probability $\left(P_{i}\right)$ : let $n_{i}(i=0 \ldots N-1)$ represent total number of stations in $i$ th class. Then a transmitted frame would collide if more than one station starts to transmit in the same timeslot. Hence, the collision probability in class $i$ would be equal to

$$
\begin{aligned}
& p_{i}=\{1-\left[\prod_{h=0}^{i-1}\left(1-\tau_{h}\right)^{n_{h}}\right] \cdot\left(1-\tau_{i}\right)^{n_{i}-1} \\
&\left.\cdot\left[\prod_{h=i+1}^{N-1}\left(1-\tau_{h}\right)^{n_{h}}\right]\right\} \cdot\left\{1-P_{\text {Channel }}\right\} .
\end{aligned}
$$

In “(3)," $P_{\text {Channel }}$ indicates the total channel's effects like noise, interference, and fading.

(ii) Successful Transmission Probability $\left(P_{s, i}\right.$ and $\left.P_{s}\right)$ : a transmission from a station belonging to class $i$ would be successful if just one station transmits at any instance of time and other stations from all classes refrain from transmission at that instance. Hence,

$$
\begin{gathered}
p_{s, i}=n_{i} \cdot \tau_{i} \cdot\left(1-\tau_{i}\right)^{n_{i}-1} \cdot\left[\prod_{h=0, h \neq i}^{N-1}\left(1-\tau_{h}\right)^{n_{h}}\right], \\
p_{s}=\sum_{i=0}^{N-1} p_{s, i}=\left(1-p_{\text {busy }}\right) \cdot \sum_{i=0}^{N-1} \frac{n_{i} \cdot \tau_{i}}{1-\tau_{i}} .
\end{gathered}
$$

Figure 2 shows all above probabilities' trend concurrently for a network of five stations.

2.2. Important Network Metrics. Before further proceed, we need to introduce two of the most important network's quantities in networks that we will extensively utilize in the next section treatments, that is, throughput and delay.

(i) Throughput ( $\left.T_{\text {hroughput }}[i]\right)$ : here, we mean from $T_{\text {hroughput }}[i](i=0 \ldots N-1)$ the normalized throughput for priority $i$ class. Also, suppose that $\sigma, T_{\text {Payload }, i}, T_{\text {Success }, i}$, and $T_{\text {Collision }, i}$ represent, respectively, slot's length, time needed to successfully transmit the average payload's length, successful transmission duration, and finally the collision's length in class $i$. Then throughput is defined as

$$
\begin{aligned}
& T_{\text {hroughput }}[i] \\
& \quad=\frac{E \text { (Payload in a slottime })}{E \text { (Length of a slottime) }} \\
& \quad=\frac{p_{s, i} \cdot T_{\text {Payload }, i}}{\left(1-p_{b}\right) \cdot \sigma+p_{s} \cdot T_{\text {Success }, i}+\left(p_{b}-p_{s}\right) \cdot T_{\text {Collision }, i}} .
\end{aligned}
$$




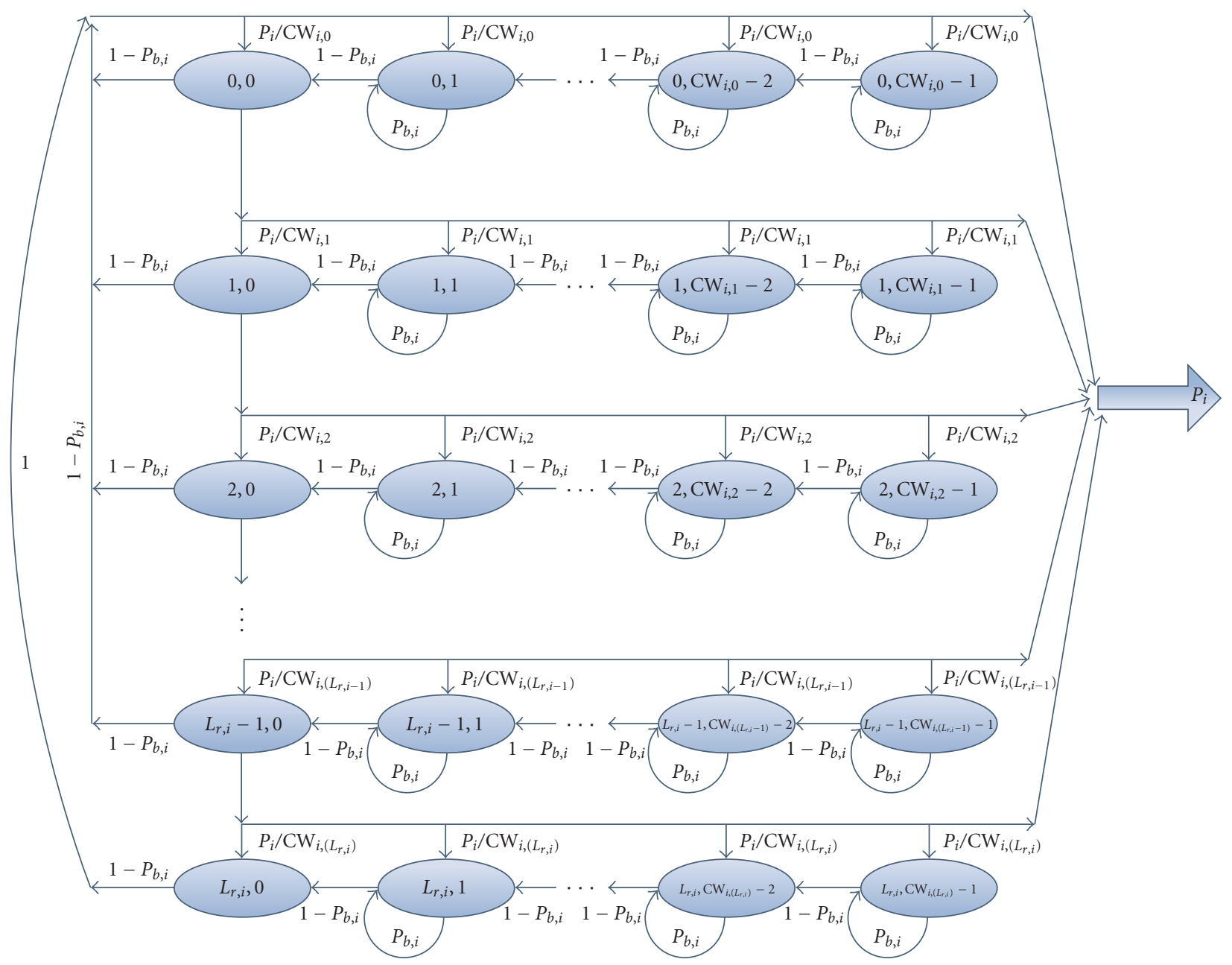

FIgURE 1: This diagram is a modified Markovian chain summarizing the back-off procedure in 802.11e networks.

(ii) Delivery delay or Service delay $\left(D_{\text {Delivery }, i}\right)$ : this metric is equal to the time duration between two successive successful transmission from a station (in class $i$ ). This metric includes propagation delay, IFS, access delay, (including collisions and back-off), and Ack's length. By applying widespread Little rule, the mean delivery delay for class $i$ is obtainable as:

$$
D_{\text {Delivery,i }}=\frac{E\left(\text { Payload }_{i}\right)}{T_{\text {hroughput }}[i]} .
$$

\section{Novel Priority Scheme Based on PDF Differentiation}

In this section, we are going to introduce a new prioritization scheme. This scheme works based on substituting Gamma distribution with legacy Uniform one that has been used in Back-off mechanism. Due to its distinctive differentiating properties, Gamma PDF seems a suitable and useful choice for being used in access granting part of network.

Although the legacy Uniform PDF, which is utilized in back-off procedure of 802.11 networks, reduces collision probability to some extent, however, the uniformity property of this PDF deeply endangers QoS issue in WLANs. This is not a complicated task to prove this claim. Actually, the key purpose of using Uniform PDF in DCF back-off mechanism was to furnish stations with strict access fairness and because there should have been no priority between stations in a 802.11 network. Nevertheless, upon emergence of 802.11e networks, this target (Fairness) was gradually vanished and replaced with prioritization. Our familiarity with mathematical analysis and higher-order statistical moments (Like Kurtosis) further confirms this fact that Uniform PDF's dispersion is too high to be suitable for differentiation purposes. This property makes it an inappropriate option especially for voice and video servicing at the same time with Best-effort services. To gain the required tools, in next subsection, we first go over few probabilistic means that are required for further proceed.

3.1. Prioritization Based on Gamma PDF. In current section's analysis, we do vastly use a probabilistic moment named Kurtosis. Hence, it is necessary to introduce it, in advance. Actually, Kurtosis is a measure of the concentration of 


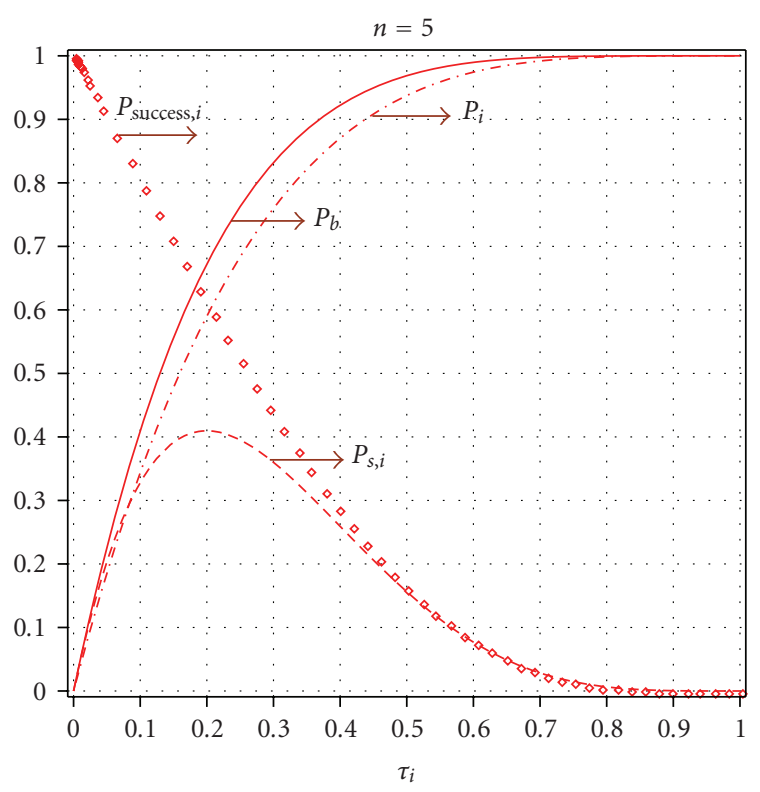

FIGURE 2: All above network probabilities in one place. These curves are plotted versus transmission probability $\left(\tau_{i}\right)$ for a network of five stations.

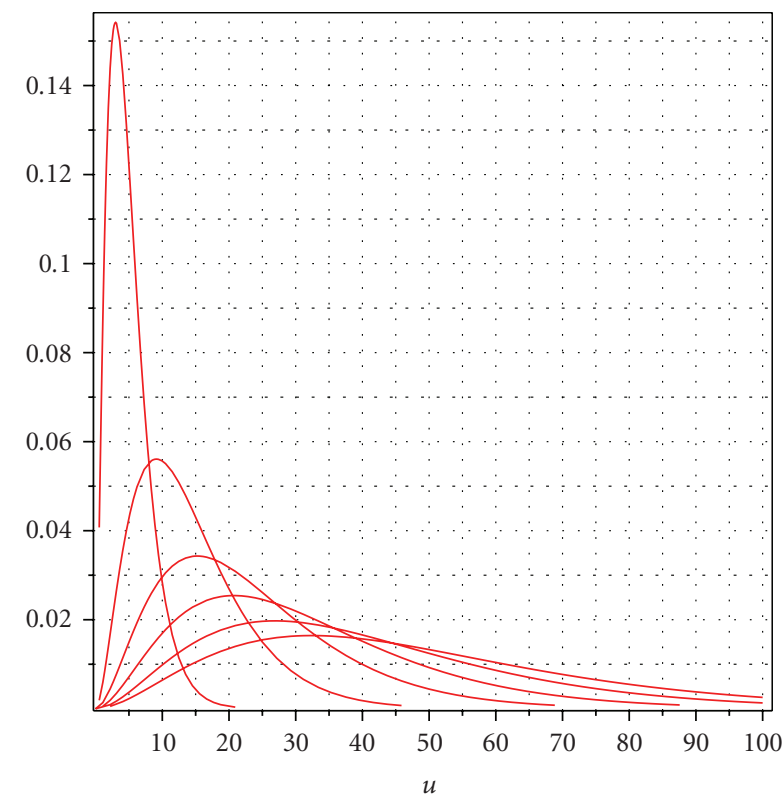

FIgURE 3: Gamma PDF curves for different parameters' values.

a distribution about its mean and larger value of this metric is equivalent to a narrower distribution and more concentration about its mean:

$$
B_{2}=\frac{m_{4}}{m_{2}^{2}} .
$$

where $m_{2}$ and $m_{4}$ are, respectively, second and forth central moments of a distribution (normal distribution has $B_{2}=3$ for all its parameters' values).

In order to improve strict QoS level in network and especially to better support delay sensitive traffics like voice and video, we apply Gamma PDF instead of Uniform one. Gamma PDF is a continuous two-parameter distribution from which the Chi-Square and Exponential PDFs are derivable. This PDF varies widely as its two parameters change and takes different shapes with distinctive characteristics. The scalability of this PDF makes it suitable for our purpose, which is setting up a wide interclass differentiation. Figure 3 shows Gamma's trend for different $b$ and $c$ values:

$$
\begin{array}{r}
\operatorname{PDF}(\operatorname{Gamma}(b, c))=\left\{\begin{array}{cc}
0 & u<0 \\
\frac{(u / b)^{c-1} \cdot e^{-u / b}}{b \cdot \gamma(c)} & u>0
\end{array}\right\}, \\
\text { where } \gamma(c)=\int_{0}^{\infty} e^{-t} \cdot t^{c-1} \cdot d t .
\end{array}
$$

The mean, Variance, and Kurtosis of Gamma PDF are

Mean $=b \cdot c, \quad \operatorname{Var}=b^{2} \cdot c, \quad$ Kurtosis $=3 \cdot \frac{(2+c)}{c}$.

As it is evident, the Kurtosis of Gamma PDF only depends on shape parameter $(c)$.

This PDF has the property to furnish higher-priority classes with PDF of higher kurtosis and lower-priority classes with lower kurtosis. Therefore, the higher-priority class encounters with lower collision probability and hence enjoys higher successful transmission probability. At the same time, the lower-priority class would have a PDF with lower Kurtosis value and so it gains higher freedom to choose diverse back-off values. Therefore, both classes observe improvements in their performance.

According to (9), As the scale parameter in Gamma increases from 0 to $\infty$, the kurtosis varies reversely from $\infty$ to 3. The more $c$ value results in less $B_{2}$; so at $c=\infty$ the Gamma distribution becomes a Normal one with $B_{2}=3$. By establishing a suitable relationship between $c$ and one of the differentiation parameters in network (like $\mathrm{CW}_{\min }$ ) in a way that higher-priority class enjoys lower $c$ and vice versa, the enjoyable results will be achieved.

The interesting fact about the use of Uniform $(a, b)$ PDF in 802.11e networks is that it always enjoys Kurtosis $=1.8$ regardless of its $b$ and a border's values. As a result of this fact, not only Kurtosis $<2$ is a low value for making strict differentiation between different traffic classes, but also being constant for all values of RV and all $a$ and $b$ values is a great cause of poor performance of Uniform PDF (when used in Back-off mechanism).

The Gamma distribution will gain better performance for lower values of $c$; for instance, for $c=1$ the Gamma PDF becomes an Exponential one with better QoS support than $c>1$. This fact will be shown in simulation results of next section.

Furthermore, Gamma distribution has another interactive attribute in comparison with Uniform's one: existence of two degrees of freedom in Gamma $(b, c)$ instead of one degree of freedom in Uniform ( $b$ - $a$ ) helps in furnishing network with two different kinds of differentiation: (1) interclass 


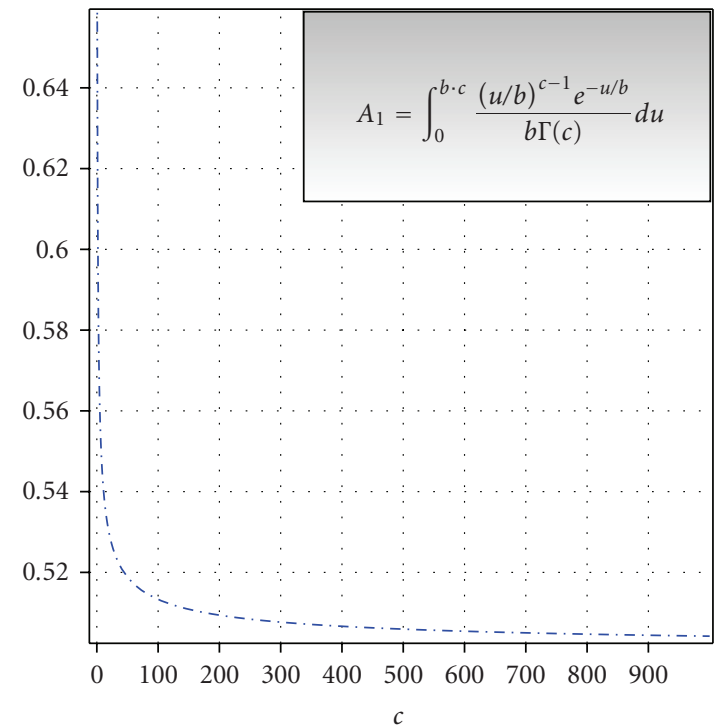

(a)

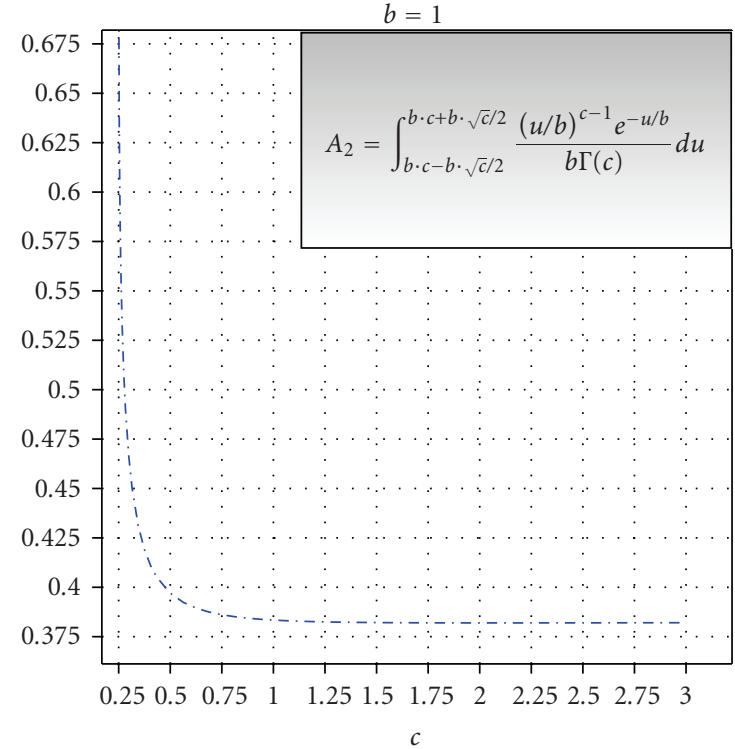

(b)

Figure 4: $A_{1}$ and $A_{2}$ curves versus $c$ parameter. As $c$ value increases from 0 to $\infty, A_{1}$ reduces from 1 to 0.5 , and $A_{2}$ reduces from 1 to 0.38 .

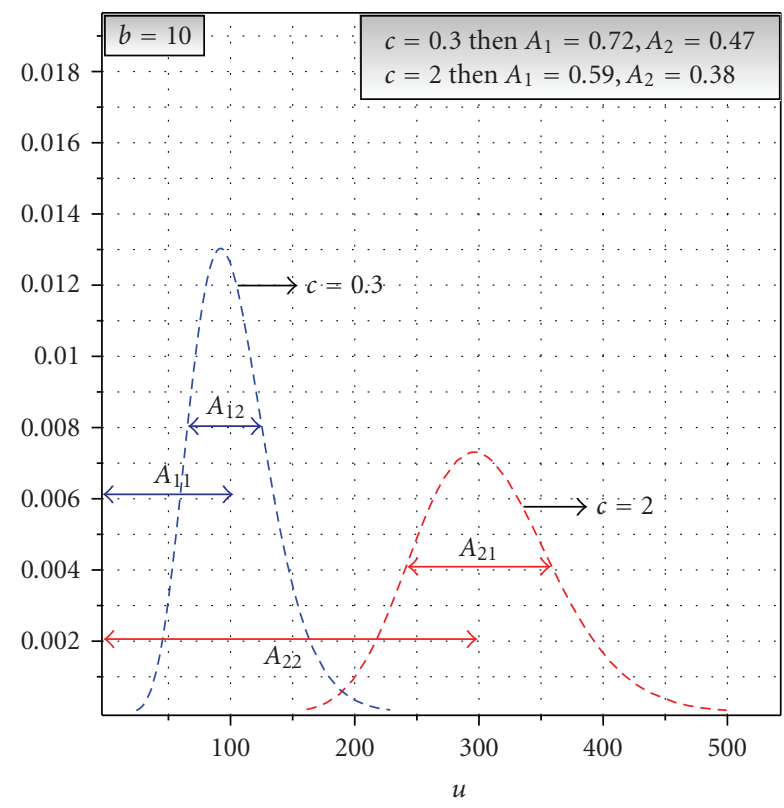

FIGURE 5: This plot illustrates how increasing $c$ directly affects $A_{1}$ and $A_{2}$ integrals and hence stretches its shape. Both PDFs plotted for constant $b=10$.

differentiation and (2) intraclass differentiation. In interclass differentiation, diverse traffic classes are to be served with different PDFs while in Intra-class differentiation, one frame in different transmission stages (after unsuccessful transmissions) is to be served with different PDFs. Uniform PDF with one degree of freedom $(b-a)$ treats these two distinct differentiations as one and hence QoS provisioning is endangered.
To better illustrate above explanations, we talk a little probability mass transfer in Gamma $(b, c)$ PDF that happens when $b$ and $c$ change. Below we introduce two important probabilistic parameters in Gamma PDF that take important role in our analysis.

(i) Cumulative probability up to Mean:

$$
A_{1}=\int_{0}^{\text {Mean }=b \cdot c} \operatorname{Gamma}(b, c, u) \cdot d u .
$$

(ii) Cumulative probability around mean:

$$
A_{2}=\int_{b \cdot c-b \cdot \sqrt{c / 2}}^{b \cdot c+b \cdot \sqrt{c / 2}} \operatorname{Gamma}(b, c, u) \cdot d u .
$$

The interesting fact is that, like kurtosis, this two metrics are also independent from scale parameter $(b)$ and depend only on shape parameter $(c)$.

Consider two integrals $A_{1}$ and $A_{2}$. Obvious from above equations, these two integrals' values only depend on $c$ parameter and are independent of $b$ whatsoever. As $c$ value increases from 0 to $\infty, A_{1}$ monotonically reduces from 1 to 0.5. This fact is illustrated at Figure 4(a). This means that, in a network that utilizes Gamma PDF in Back-off procedure instead of Uniform one, the higher-priority class chooses its back-off counter's value from $0<u<b \cdot c$ (Mean of Gamma $(b, c))$ interval with higher probability than the lower-priority class. Correspondingly, $A_{2}$ reduces from 1 to 0.38 (again this proves the increasing similarity of Gamma and Normal as $c$ tends to $\infty$ and $A_{2}(\operatorname{Normal}(\mu, \sigma 2))=$ 0.38292). This fact is illustrated at Figure 4(b).

These explanations emphasize on one important fact: as $c$ increases, not only the dispersion of Gamma PDF increases (the concentration about its mean decreases) but also the 


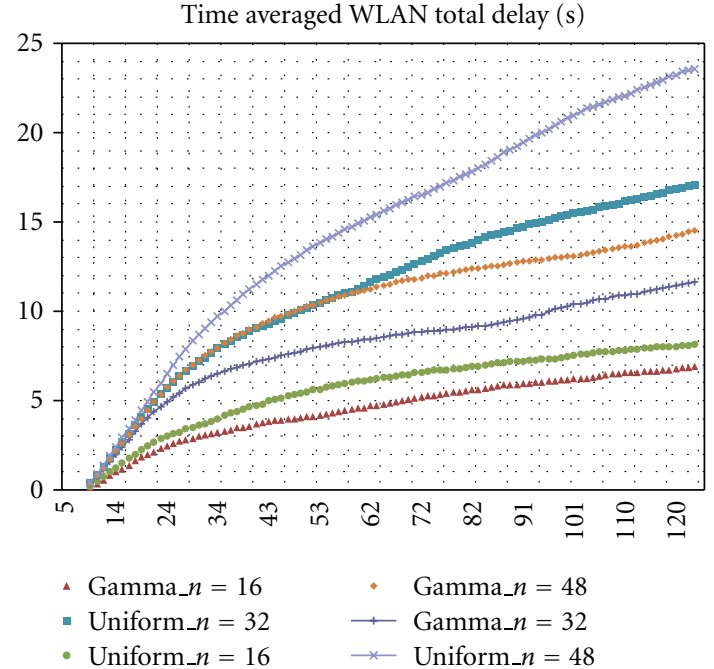

(a)

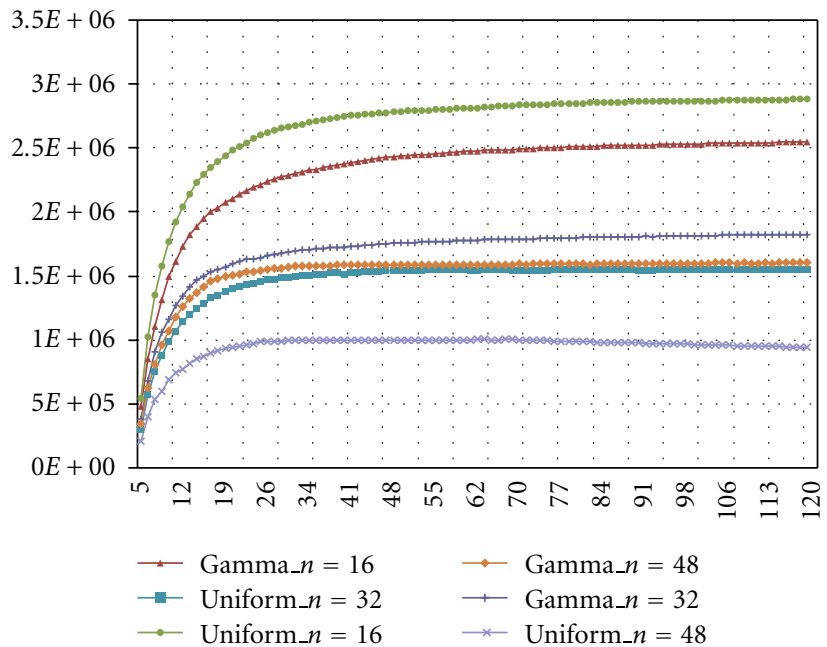

(b)

Figure 6: As illustrated, each bundle includes two simulations: one Gamma curve and one PDF curve. (right): Total network throughput comparison between Gamma and Uniform PDFs. Gamma PDF outperforms Uniform one especially for higher number of contending stations. (left): Total service delay comparison. In all scenarios, lower total delay achieves when applying Gamma instead.

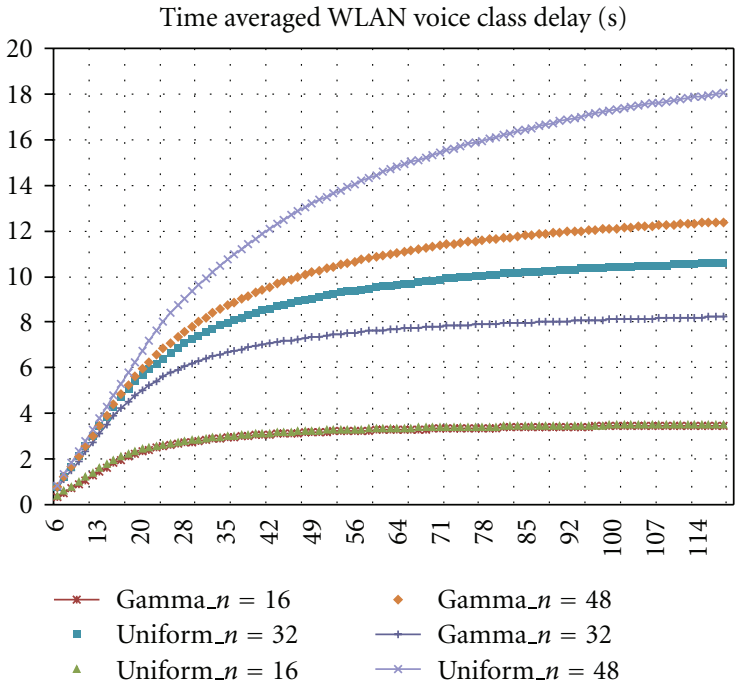

(a)

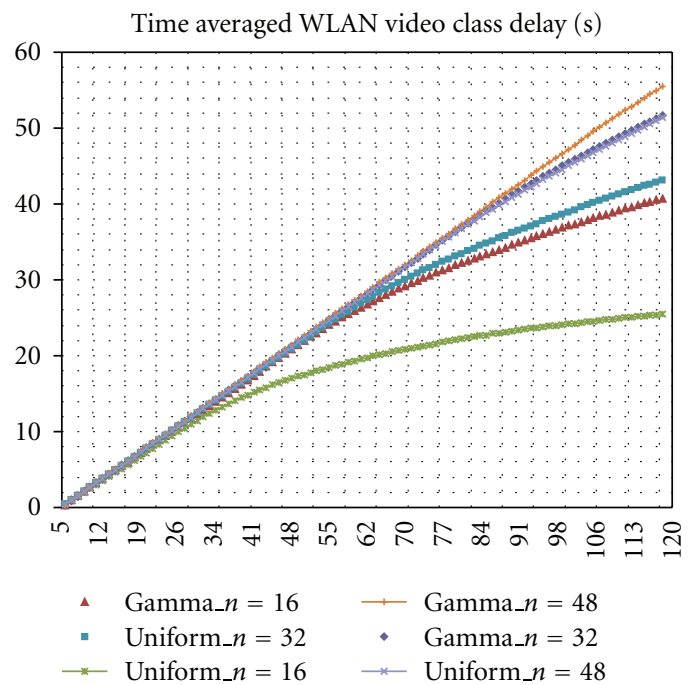

(b)

Figure 7: As illustrated, each bundle includes two simulations: one Gamma curve and one PDF curve. (right): Video class's service delay is worse in Gamma case than Uniform case. However, the difference is lessened when number of stations increases. (left): Voice class delay in Gamma Scenarios always is lower than Uniform scenarios.

mass probability transfer takes place and moves from left to right as shown in Figure 5. Again, this is evidently an advantage along QoS provisioning.

\section{Performance Evaluation}

In order to set up a correct comparison between scenarios using Uniform PDF in their back-off procedure and the ones using $\operatorname{Gamma}(b, c) \mathrm{PDF}$, it is initially mandatory to make working conditions in both scenarios similar. This means that we should identically parameterize these PDFs to get valid results. For instance, we should not set up a comparison while mean values of these two PDFs are different.

Apparently, the discrete Uniform $\left(0, \mathrm{CW}_{i, j}\right)$ has Mean = $\mathrm{CW}_{i, j} / 2$ and Variance $=\mathrm{CW}_{i, j} *\left(\mathrm{CW}_{i, j}+2\right) / 12$. To accomplish the above-mentioned task, let us equate first two moments of Gamma PDF with those of Uniform PDF. These two 


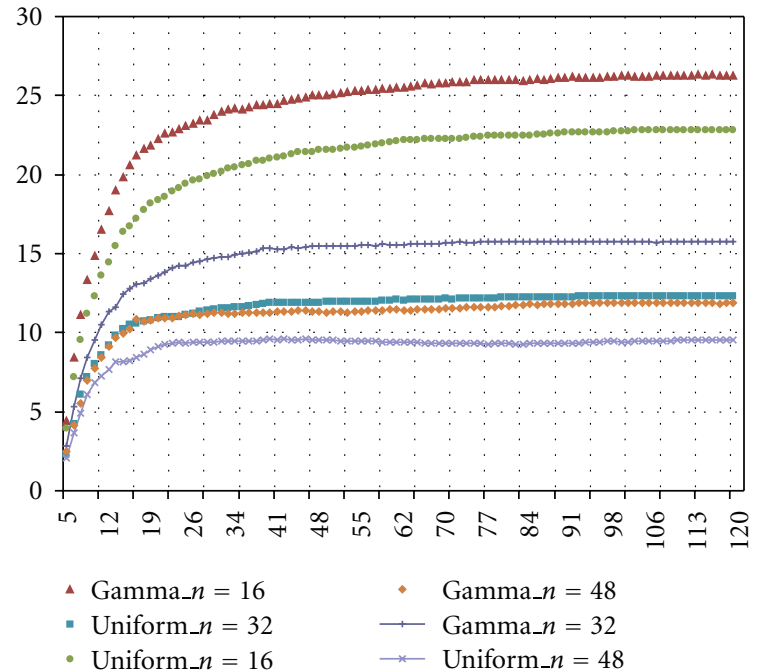

(a)

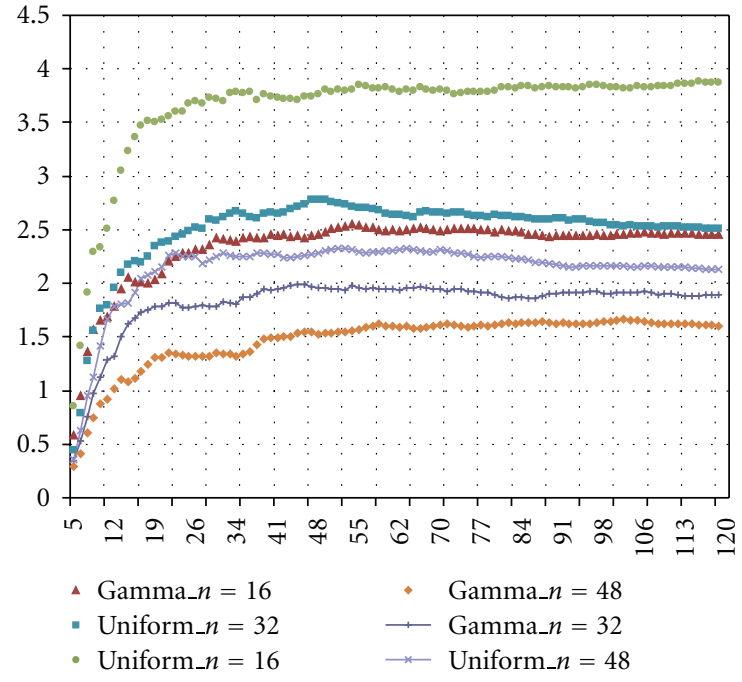

(b)

Figure 8: Transmission probability $\left(\tau_{i}\right)$ for both video and voice class. Although the trends for these classes are opposite each other (in voice class (a) Uniform PDF has always lower $\tau_{i}$ than Gamma while in video class (b) trends are reverse), but this is not a contradiction. As will be explained later both these plots vote to superiority of Gamma PDF.

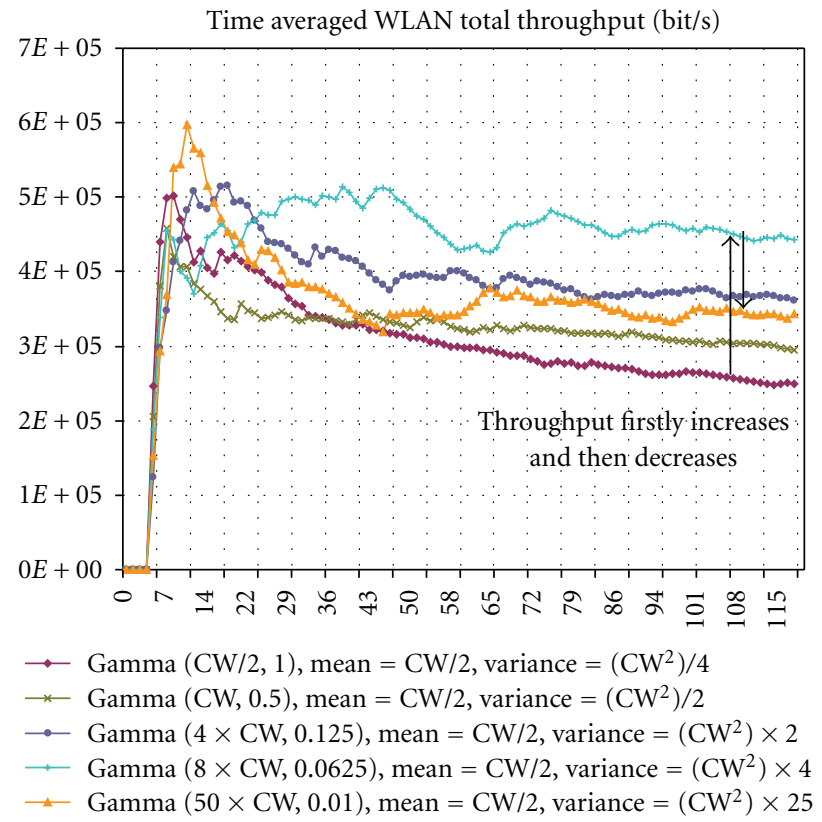

(a)

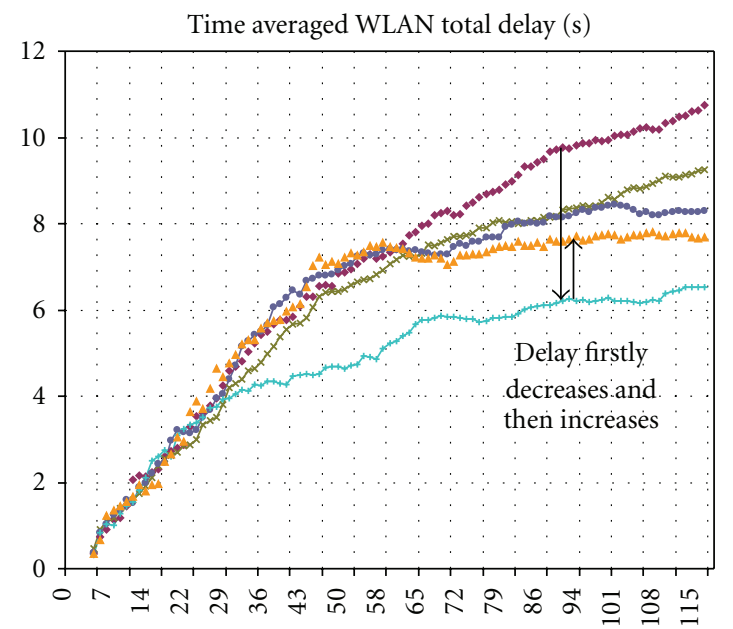

- $\operatorname{Gamma}(\mathrm{CW} / 2,1)$, mean $=\mathrm{CW} / 2$, variance $=\left(\mathrm{CW}^{2}\right) / 4$

$*$ Gamma (CW, 0.5), mean $=\mathrm{CW} / 2$, variance $=\left(\mathrm{CW}^{2}\right) / 2$

$\rightarrow$ Gamma $(4 \times \mathrm{CW}, 0.125)$, mean $=\mathrm{CW} / 2$, variance $=\left(\mathrm{CW}^{2}\right) \times 2$

$\rightarrow$ Gamma $(8 \times \mathrm{CW}, 0.0625)$, mean $=\mathrm{CW} / 2$, variance $=\left(\mathrm{CW}^{2}\right) \times 4$

$\triangle \quad$ Gamma $(50 \times \mathrm{CW}, 0.01)$, mean $=\mathrm{CW} / 2$, variance $=\left(\mathrm{CW}^{2}\right) \times 25$

Figure 9: Total throughput and total delay for five different scenarios with the same Gamma PDF mean but different variances $(n=24)$. With variance increment, Intra-class contention takes place of Inter-class contention. (right): Total delay firstly decreases (gets better) and after a limit increases (gets worse). (left), Total throughput firstly increases (gets better) and after a limit decreases (gets worse).

equations result in corresponding $c$ and $b$. Hence,

$$
\left\{\begin{array}{c}
b \cdot c=\frac{\mathrm{CW}_{i, j}}{2} \\
b^{2} \cdot c=\frac{\mathrm{CW}_{i, j} \cdot\left(\mathrm{CW}_{i, j}+2\right)}{12}
\end{array}\right\} \longrightarrow\left\{\begin{array}{l}
b=\frac{\mathrm{CW}_{i, j}+2}{6} \\
c=\frac{3 \cdot \mathrm{CW}_{i, j}}{\mathrm{CW}_{i, j}+2}
\end{array}\right\} .
$$

Evidently, Gamma(b $=\left(\mathrm{CW}_{i, j}+2\right) / 6, c=(3$. $\left.\left.\mathrm{CW}_{i, j}\right) /\left(\mathrm{CW}_{i, j}+2\right)\right)$ depends only on one parameter, which is the back-off window's length at jth stage $\left(\mathrm{CW}_{i, j}\right)$. Due to monotonically increasing trend of Mean and variance (with $\mathrm{CW}_{i, j}$ ), the desirable behavior is achieved. We study three different scenarios on this basis. The distinguishing parameter in these scenarios will be the number of stations 


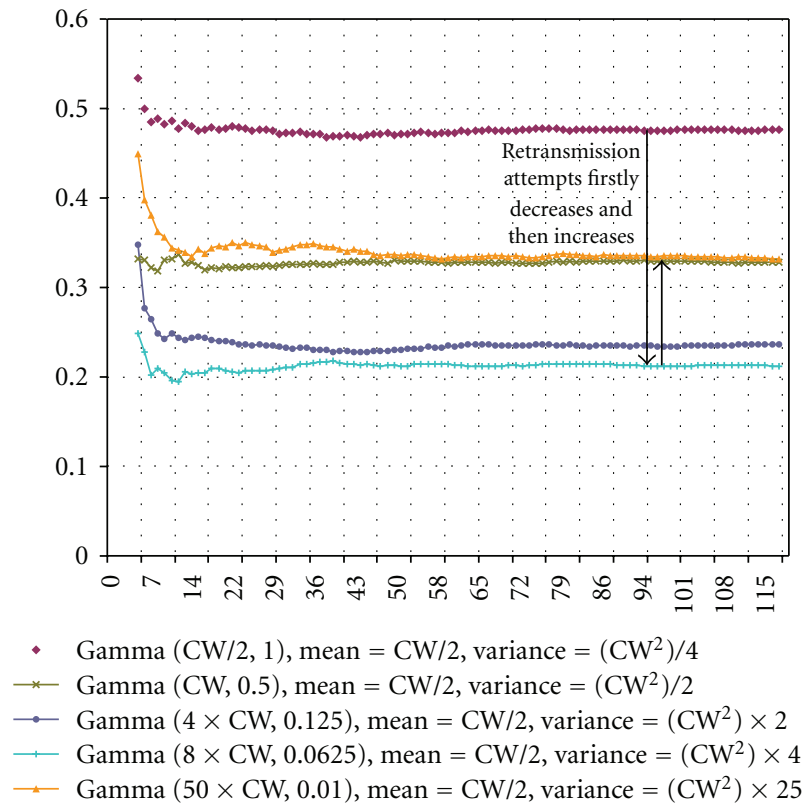

Figure 10: Mean number of retransmission attempts for five different scenarios with the same Gamma PDF mean but different variances $(n=24)$. This quantity is a direct sign of collision probability in network. In this figure, with variance increment, firstly the mean retransmission attempt decreases (gets better) and then increases (gets worse).

(n). The EDCF and common parameters that are to be applied to this set of scenarios are listed in Tables 1 and 2.

We go over three different simulation scenarios with $n=16,24,48$, in each scenario four traffic classes contend for channel access (hence $n_{i}=4,6,12$, resp.). At each scenario, two simulations are established, one with Uniform PDF and another with Gamma PDF. Several parameters are evaluated and compared in each scenario, for example, average total delay, average voice and video class service delays, throughput, and finally the transmission probability.

Figures 6, 7, and 8 better illustrate the situation. As we see, the more priority class (Voice) enjoys higher QoS than lower priority class (Video) when using Gamma PDF instead of Uniform one. Especially, total delay improves (Reduces) with Gamma PDF and the distinction becomes larger as the number of stations increases.

To better illustrate tradeoff procedure between classes (as PDF's variance increases), we plot throughput and transmission probability separately for voice, video, and best effort classes in Figures 11 and 12. As far as background class showed the same trend as best effort class, we avoid mentioning it here.

This fact that larger numbers of stations shift benefits toward using Gamma PDF stems from its lowered collision probability. As Background and Best effort traffic classes exhibited the same trends as voice class (improved in Gamma scenarios), we intentionally avoid plotting them at this place.
Thus far, we have verified superiorities of using Gamma PDF compared with Uniform one, in scenarios that mean and variance are forced to be the same. Thereafter, we focus on scenarios in which means are the same (as it is mandatory for valid comparisons) but we change variance and observe the results. The observations reveal that, as variance increases, the total delay decreases, and the total throughput increases up to a limit. After this limit, both trends are reversed with more variance increments. Interestingly, trade-off takes place between classes so that higherpriority class seizes bandwidth from lower-priority class when variance increases more and more. Therefore, collision probability decreases. The underneath reason for the lowered delay is also in exact relationship with collision probability; the less collisions happen, the less retransmission attempts take place, and consequently the lower delay would be obtained.

Fundamentally, Increasing the PDF's variance intensifies Intra-class contention and decreases Inter-class contention. Intra-class contentions are those that happen between stations in the same class, but Inter-class contention happen between stations belonging to different classes. By increasing PDF's variance more and more, after a while, the Intraclass contention becomes dominant colliding factor; hence, after passing a limit the trends are reversed and total delay increases while total throughput decreases. These explanations illustrated at Figures 9 and 10. In these sets of scenarios $n=24$.

To better illustrate tradeoff procedure between classes (as PDF's variance increases), we plot throughput and transmission probability separately for voice, video, and best effort classes in Figures 11 and 12. As far as, Background class showed the same trend as best effort class we avoid mentioning it here.

As throughput is directly engaged with transmission probability, we expect a correspondence between these two parameters. As an example, let us take a look at voice and video classes transmission probabilities.

As it is obvious, the transmission probabilities of these two classes vary in opposite directions although throughputs in both classes (Figure 12) vary in the same directions. This should not seem confusing since Figure 13 can best explain the reason.

Figure 13 shows that both throughput and successful transmission probability have maximum in $0<\tau \approx 1 /(n+$ $1)<1$. The descending/ascending behavior of these two metrics as a function of $\tau$ has divided them into two distinct regions and the separation point is this maximum. Taking a deeper look on this plot will solve the confusion. In EDCF, the higher-priority class (voice) always survives in descending part of curves (region 1) while other classes survive in its ascending part (region 2). As PDF's variance increases, voice class's $\tau$ decreases, and other classes' $\tau$ increase. This leads to higher throughput in voice class because in Region 1 the trend is monotonically descending. At the other side, ascending behavior of plot in region 2 leads to higher throughput in other classes when $\tau$ increases. Due to the same fact, transmission probability in voice class is always higher than other classes. 


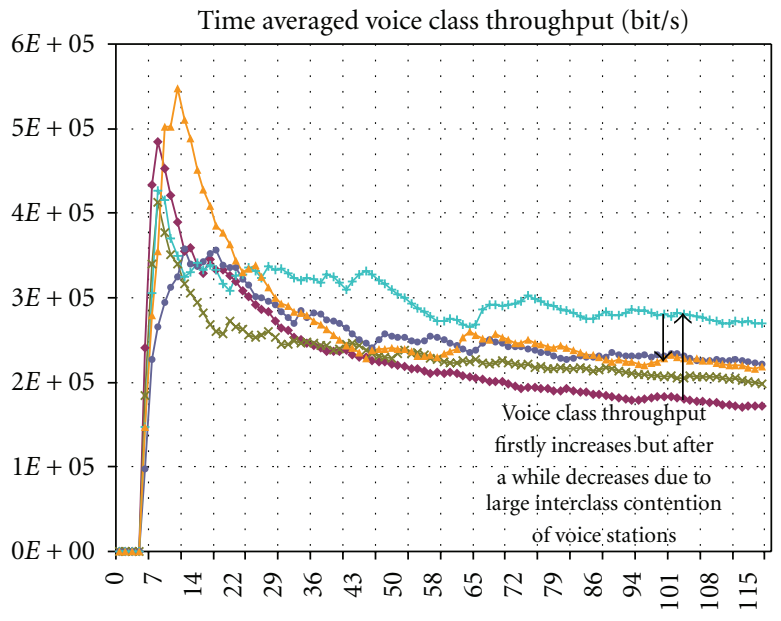

(a)

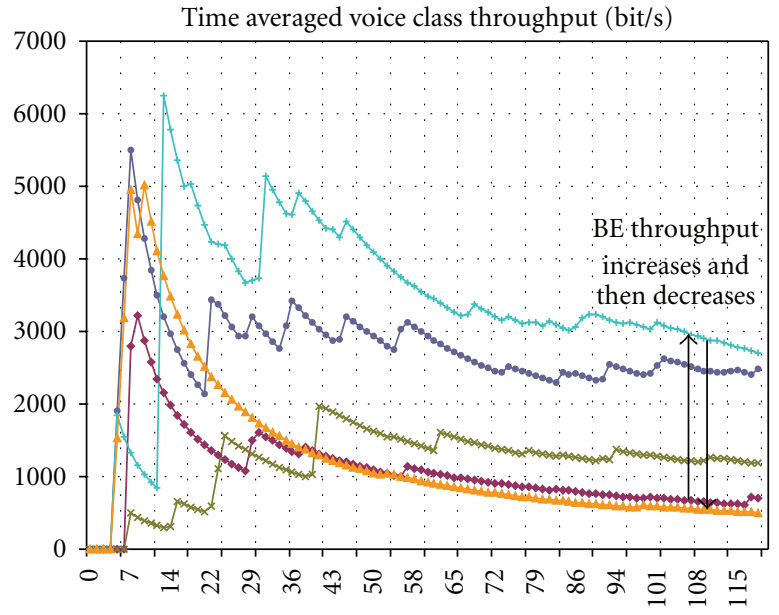

(b)

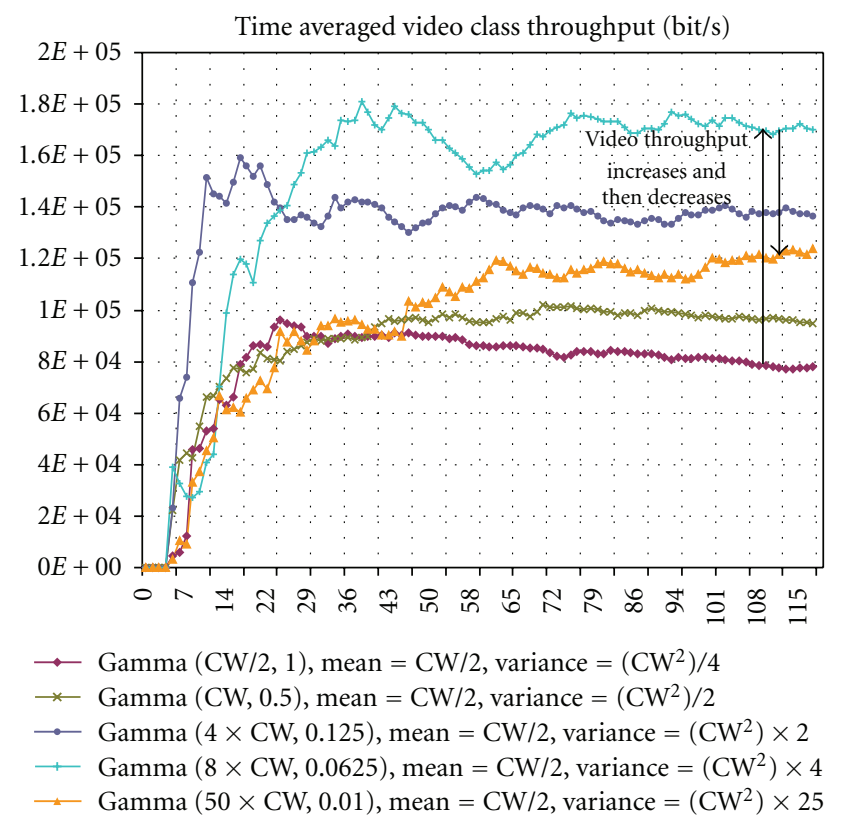

(c)

FIGURE 11: Throughput trends of voice, video, and best effort classes for five different scenarios $(n=24)$. In all these three classes, with PDF variance increment, throughput firstly increases (gets better) and after a threshold decreased (gets worse).

TABLE 1: EDCF parameter set.

\begin{tabular}{lcccc}
\hline & Voice $(\mathrm{VO})$ & Video $(\mathrm{VI})$ & Best Effort (BE) & Background (BG) \\
\hline $\mathrm{CW}_{\min }$ & 3 & 7 & 31 & 63 \\
$\mathrm{CW}_{\max }$ & 7 & 63 & 1023 & 8192 \\
$\mathrm{AIFS}$ & 2 & 2 & 2 & 2 \\
$\mathrm{TXOP}$ & $3264 \mu \mathrm{s}$ & $3264 \mu \mathrm{s}$ & $3264 \mu \mathrm{s}$ & $3264 \mu \mathrm{s}$ \\
\hline
\end{tabular}

TABLE 2: Common parameter set.

\begin{tabular}{lccccc}
\hline PHY & Transmitted Power (W) & RTS Used & Buffer Size (bit) & Traffic Inter arrival Time (S) & Packet Size (Byte) \\
\hline DSSS-11 Mbit/s & 0.005 & None & 256000 & 0.005 & 1024 \\
\hline
\end{tabular}




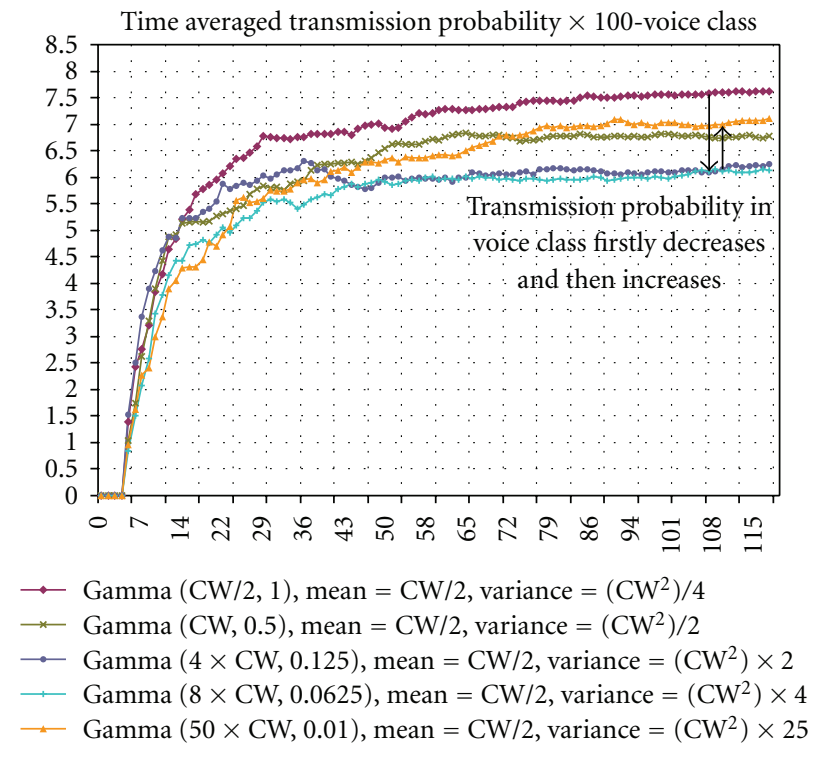

(a)

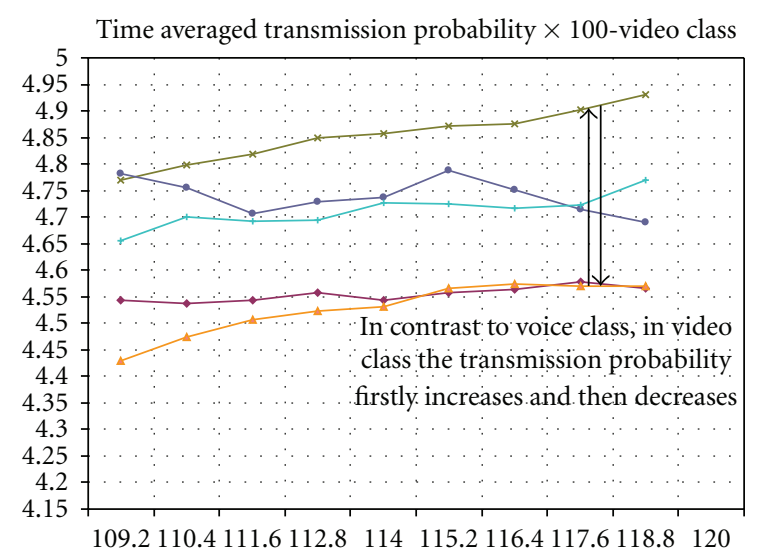

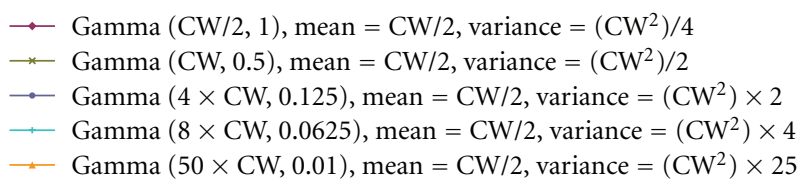

(b)

FIgURE 12: Transmission probabilities of voice and video classes for five different scenarios $(n=24)$. With variance increment, Intra-class contention takes place of Inter-class contention. (right): $\tau_{i}$ for video class increases and then decreases. (left): In contrast to video class, in voice class $\tau_{i}$ firstly decreases and then increases.

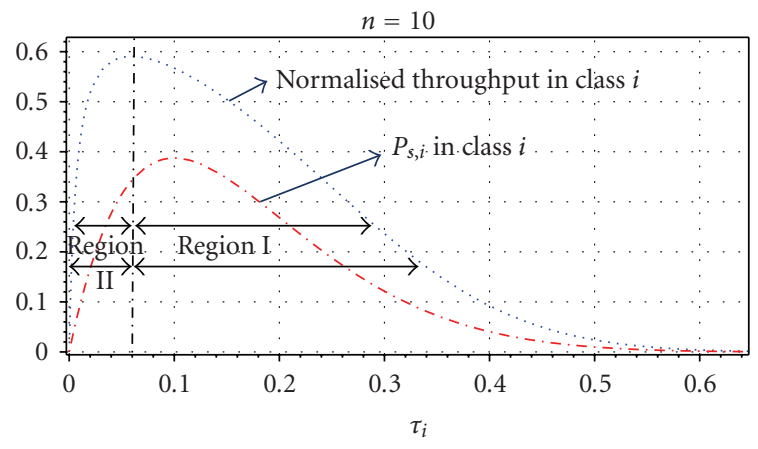

FIgURE 13: Normalized throughput and successful transmission probability versus transmission probability $\left(\tau_{i}\right)$ for a network of $n=10$. When several classes contend for channel access, the priority class (voice, here) survives in Region1 while other classes are sentenced to be in Region 2.

\section{Comparison between Different PDFs}

At last, we summarize this article by making a comparison between service delays when different well-known probability density functions are applied on DCF. Here, we compare six scenarios with each other. The applied PDFs are Uniform, Geometric, Pareto, Exponential, Poisson, and Gamma. It is to be noted that variance and mean are the same in all these scenarios to have a valid comparison. Figure 14 illustrates this situation. It should be emphasized that, as these curves were plotted in time average mode filter of OPNET simulator, we should only consider values at simulation ending time $(2 \mathrm{~m}=60 \mathrm{~s})$ of Figure 14. Due to the nature of this filtering in OPNET, it only shows the real mean value of each curve

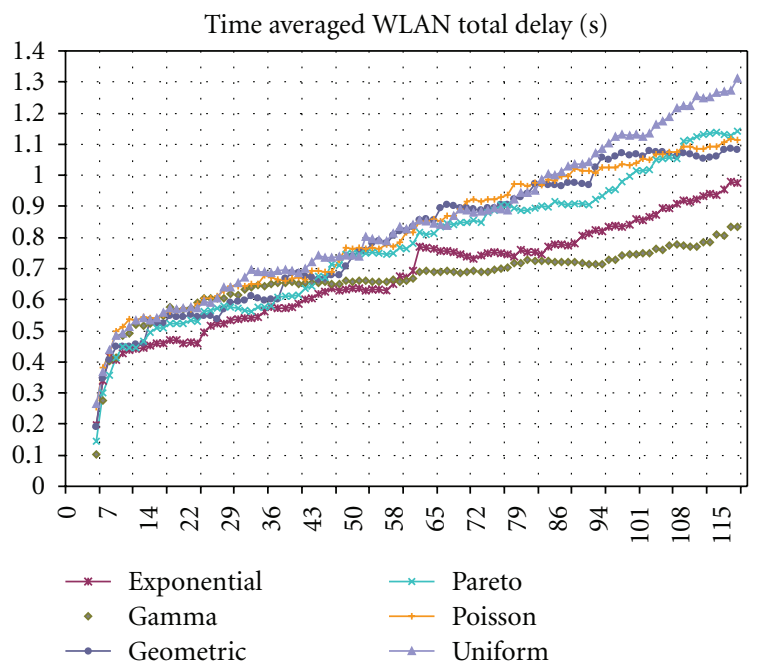

Figure 14: Total delay trends for six different well-known PDFs that implemented instead of Uniform PDF. The Gamma PDF outperforms Uniform PDF with near 65\% delay reduction. As Exponential PDF is an especial case of Gamma PDF with $c=1$, it is ranked as the second superior PDF.

at simulation ending time. Therefore, Gamma outperforms others and Uniform shows the worst behavior.

\section{Conclusion}

In this study, we proposed a new prioritization scheme that is based on PDF differentiation. In this scheme, we applied Gamma PDF in place of Uniform one. The rationale 
behind this idea is directly related to especial characteristics of Gamma PDF that it inherently possesses and makes it suitable for well-differentiating purposes. We proved this claim with the aid of comprehensive probabilistic explanations and analysis. Then we established extensive simulation runs to confirm the suitability of this scheme. Sufficient set of metrics were put under investigation like delay, throughput, retransmission attempt, and transmission probability. All results decisively confirmed that our scheme outperforms legacy EDCF.

In [27], we proposed a different QoS improving method named $\mathrm{CW}_{\min }$-ATM. To better support QoS, instead of using differentiation, we adaptively tune $\mathrm{CW}_{\min }$ after each transmission based on getting instantaneous feedback from channel's load. We showed that it is possible to combine our proposed differentiation scheme with $\mathrm{CW}_{\min }$-ATM in order to achieve better QoS in 802.11e networks.

\section{References}

[1] IEEE 802.11 WG, "Part 11: Wireless LAN Medium Access Control (MAC) and Physical Layer (PHY) Specification," 1999.

[2] IEEE 802.11a WG, "Part 11: High-Speed Physical Layer in the 5 GHz Band," 1999.

[3] A. S. Tanenbaum, Computer Networks, Prentice Hall, Upper Saddle River, NJ, USA, 4th edition, 2002.

[4] G. Bianchi, "Performance analysis of the IEEE 802.11 distributed coordination function," IEEE Journal on Selected Areas in Communications, vol. 18, no. 3, pp. 535-547, 2000.

[5] E. Ziouva and T. Antonakopoulos, "CSMA/CA performance under high traffic conditions: throughput and delay analysis," Computer Communications, vol. 25, no. 3, pp. 313-321, 2002.

[6] H. Wu, Y. Peng, K. Long, S. Cheng, and J. Ma, "Performance of reliable transport protocol over IEEE 802.11 wireless LAN: analysis and enhancement," in Proceedings of the IEEE Information Communications (INFOCOM '02), vol. 2, pp. 599-607, New York, NY, USA, 2002.

[7] Y. Xiao and J. Rosdahl, "Throughput and delay limits of IEEE 802.11," IEEE Communications Letters, vol. 6, no. 8, pp. 355$357,2002$.

[8] T. S. Ho and K. C. Chen, "Performance evaluation and enhancement of the CSMA/CA MAC protocol for 802.11 wireless LAN's," in Proceedings of the IEEE Personal Indoor and Mobile Radio Communications (PIMRC'96), pp. 392-396, Taipei, Taiwan, October 1996.

[9] B. Bing and R. Subramanian, "A novel technique for quantitative performance evaluation of wireless LANs," Computer Communications, vol. 21, no. 9, pp. 833-838, 1998.

[10] M. S. Chhaya and S. Gupta, "Performance modeling of asynchronous data transfer methods of IEEE 802.11 MAC protocol," Wireless Networks, vol. 3, no. 3, pp. 217-234, 1997.

[11] K.-C. Huang and K-C. Chen, "Interference analysis of nonpersistent CSMA with hidden terminals in multicell wireless data networks," in Proceedings of the IEEE International Symposium on Personal, Indoor and Mobile Radio Communications (PIMRC '95), vol. 2, pp. 907-911, Toronto, Canada, 1995.

[12] F. Calì, M. Conti, and E. Gregori, "Dynamic tuning of the IEEE 802.11 protocol to achieve a theoretical throughput limit," IEEE/ACM Transactions on Networking, vol. 8, no. 6, pp. 785799, 2000.
[13] F. Calì, M. Conti, and E. Gregori, "IEEE 802.11 protocol: design and performance evaluation of an adaptive back-off mechanism," IEEE Journal on Selected Areas in Communications, vol. 18, no. 19, pp. 1774-1786, 2000.

[14] Y. C. Tay and K. C. Chua, "A capacity analysis for the IEEE 802.11 MAC protocol," Wireless Networks, vol. 7, no. 2, pp. 159-171, 2001.

[15] Y. Xiao and J. Rosdahl, "A performance analysis of IEEE 802.11a Wireless LAN," in Proceedings of the Systemic, Cybernetics, and Informatics (SCI '02), pp. 243-248, Orlando, Fla, USA, 2002.

[16] J. Deng and R.-S. Chang, "A priority scheme for IEEE 802.11 DGF access method," IEICE Transactions on Communications, vol. E82-B, no. 1, pp. 96-102, 1999.

[17] Y. Xiao, "A simple and effective priority scheme for IEEE 802.11," IEEE Communications Letters, vol. 7, no. 2, pp. 70-72, 2003.

[18] A. Veres, A. T. Campbell, M. Barry, and L.-H. Sun, "Supporting service differentiation in wireless packet networks using distributed control," IEEE Journal on Selected Areas in Communications, vol. 19, no. 10, pp. 2081-2093, 2001.

[19] I. Aad and C. Castelluccia, "Differentiation mechanisms for IEEE 802.11," in Proceedings of the IEEE Information Communications (INFOCOM '01), vol. 1, pp. 209-218, Anchorage, Alaska, USA, 2001.

[20] X. Pallot and L. E. Miller, "Implementing message priority policies over an 802.11 based mobile ad hoc network," in Proceedings of the IEEE Military Communications Conference (MILCOM '01), vol. 2, pp. 860-864, McLean, Va, USA, 2001.

[21] S. Mangold, S. Choi, P. May, O. Kein, G. Hiertz, and L. Stibor, "IEEE 802.11e wireless LAN for quality of service," in Proceedings of European Wireless, pp. 32-39, Florence, Italy, 2002.

[22] IEEE 802.11e WG, "Medium Access Control (MAC) Enhancements for Quality of Service," IEEE 802.11e/D2.0, November 2001.

[23] Y. Xiao, "IEEE 802.11 E: QoS provisioning at the MAC layer," IEEE Wireless Communications, vol. 11, no. 3, pp. 72-79, 2004.

[24] Y. Xiao, H. Li, and S. Choi, "Protection and guarantee for voice and video traffic in IEEE 802.11e wireless LANs," in Proceedings of the IEEE Information Communications (INFOCOM '04), vol. 3, pp. 2152-2162, Hong Kong, 2004.

[25] G. Bianchi and I. Tinnirello, "Analysis of priority mechanisms based on differentiated inter frame spacing in CSMA-CA," in Proceedings of the IEEE Vehicular Technology Conference (VTC '03), vol. 58, pp. 1401-1405, Orlando, Fla, USA, 2003.

[26] J. Zhao, Z. Guo, Q. Zhang, and W. Zhu, "Performance study of MAC for service differentiation in IEEE 802.11," in Proceedings of the IEEE Global Telecommunications Conference (GLOBECOM '02), vol. 1, pp. 778-782, Taipei, Taiwan, 2002.

[27] N. Tadayon and S. Zokaei, "Introducing an adaptive method to tune initial back-off window (CWmin -ATM) in IEEE 802.11 wireless networks," submitted to EURASIP Journal on Wireless Communications and Networking. 

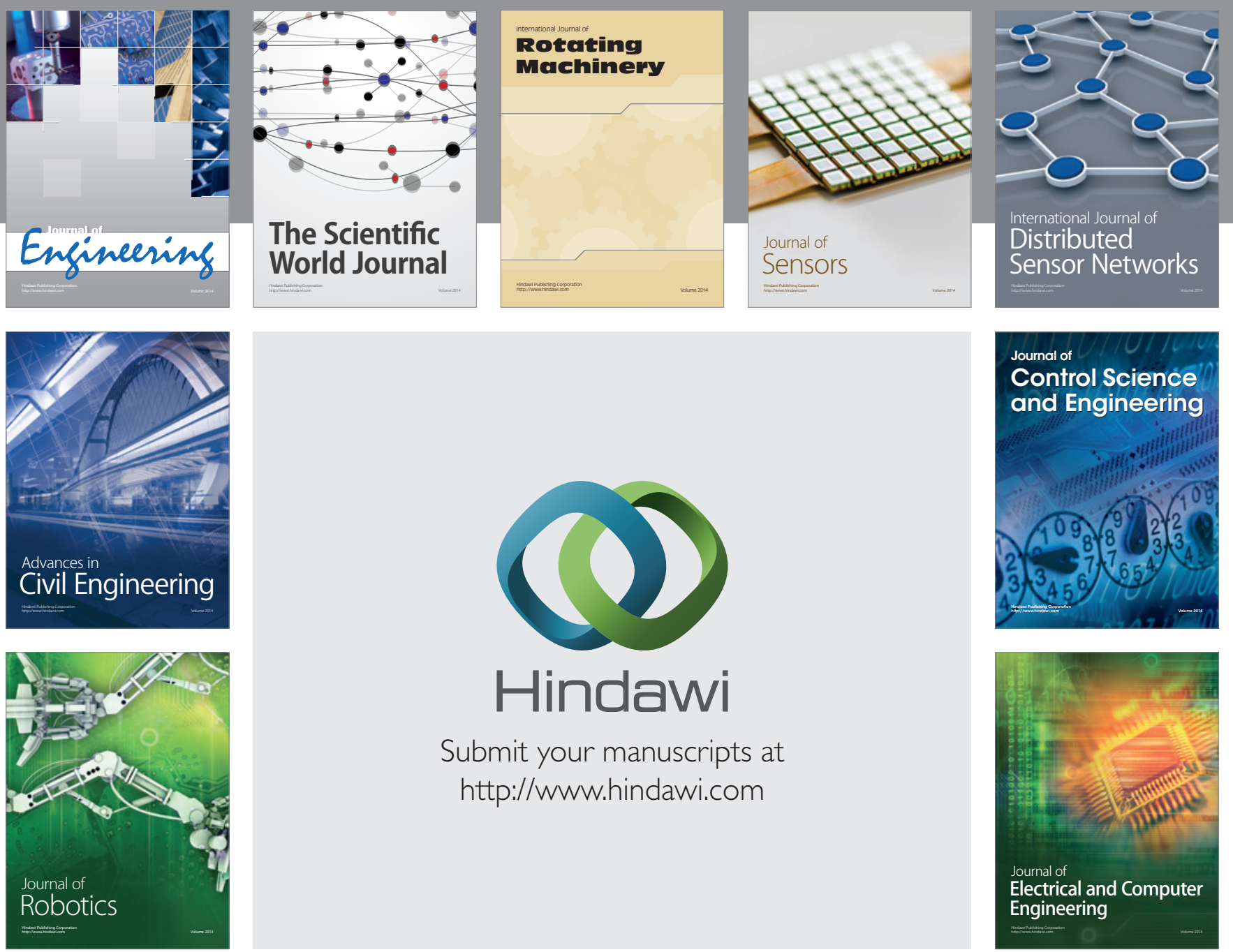

Submit your manuscripts at

http://www.hindawi.com
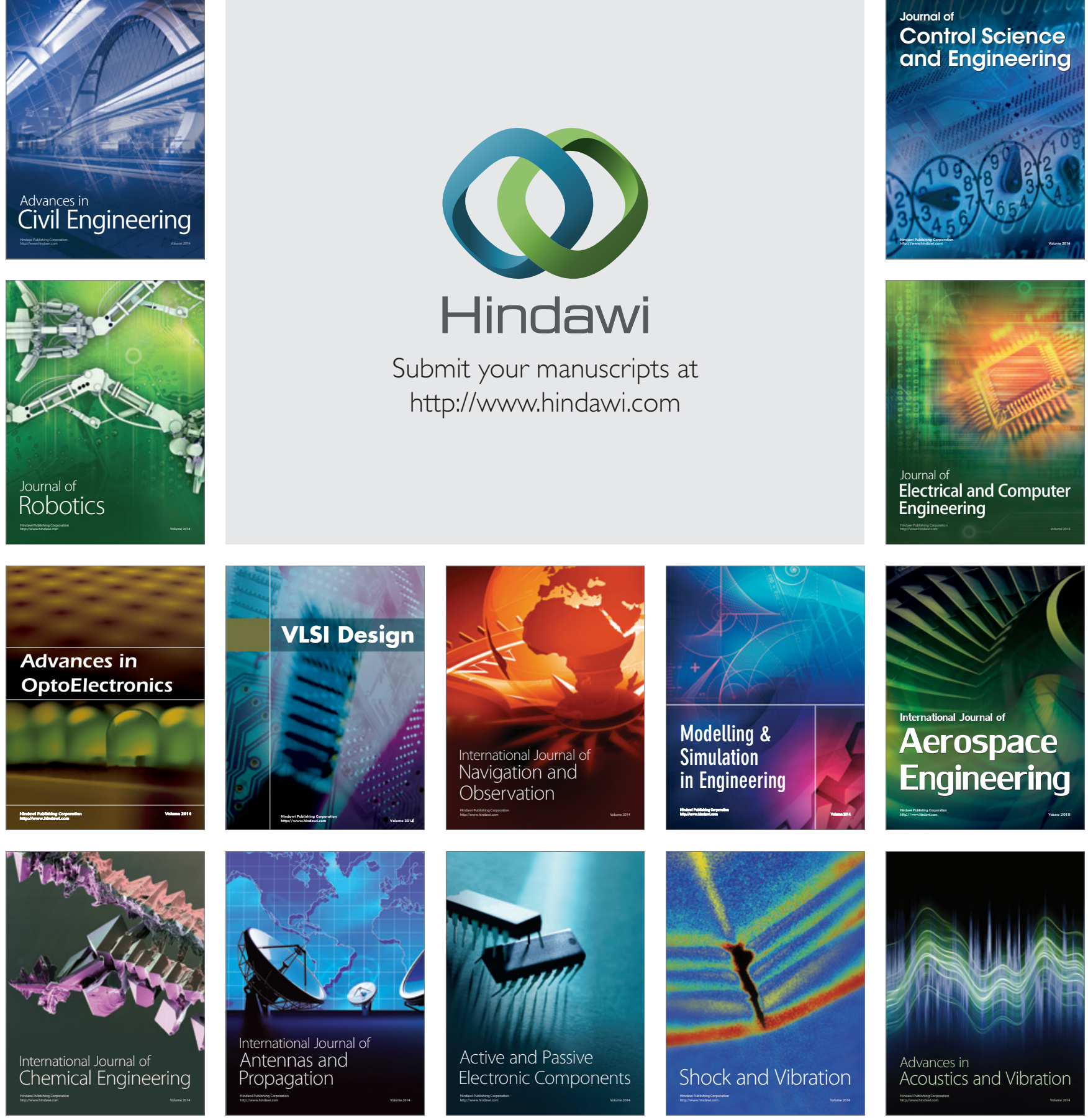